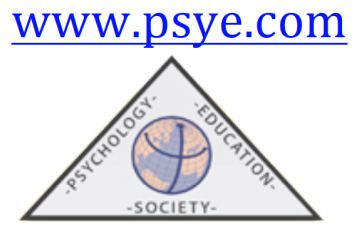

\title{
Resiliencia, optimismo y estrategias de afrontamiento en estudiantes de Ciencias de la Educación
}

\author{
Carmen María VIZOSO GÓMEZ
}

Universidad de León, España

(Recibido el 24 de Enero de 2019, Aceptado el 15 de Mayo de 2019)

\begin{abstract}
RESUMEN: En el presente trabajo se analiza la resiliencia, el optimismo-pesimismo y las estrategias activas de afrontamiento al estrés. Se evaluó a una muestra formada por 450 estudiantes de Ciencias de la Educación en la Universidad de León (España). Se administró la escala de resiliencia 10-item CDRISC, el test de optimismo LOT-R y la escala de afrontamiento activo del inventario de estrategias de afrontamiento (CSI), todos en su versión en español. Los resultados obtenidos revelaron que existen diferencias de género en cuanto a las puntuaciones en resiliencia, optimismo y en algunas estrategias de afrontamiento. Además, se encontraron relaciones significativas y positivas entre la resiliencia y el optimismo-pesimismo, así como entre la resiliencia y la mayoría de las estrategias de afrontamiento. Por último, los resultados muestran que tanto el optimismo como la estrategia de afrontamiento centrada en la resolución de problemas predicen la resiliencia en los estudiantes de Ciencias de la Educación. Por último, se propone la utilidad de los resultados para el diseño de programas de intervención para la formación de futuros educadores.
\end{abstract}

Palabras clave: Resiliencia, afrontamiento, optimismo, universitarios, educadores

\section{Resilience, optimism and coping strategies among students of Educational Sciences}

\begin{abstract}
This work analyzes resilience, optimism-pessimism and active coping strategies. A sample of 450 students of Educational Sciences at the University of León (Spain) was evaluated. The Spanish version of the 10-item CD-RISC, LOT-R and the Active Coping Scale from CSI were administered. The results revealed that there are gender differences in terms of resilience, optimism and some coping strategies. In addition, significant and positive relationships were found between resilience and optimism-pessimism, as well as between resilience and most of the coping strategies. Finally, the results show that both optimism and coping strategy focused on problem solving predict resilience in the students of Education Sciences. Finally, the utility of these results for designing intervention programs in the training of future educators is proposed.
\end{abstract}

Keywords: Resilience, coping, optimism, university students, educators

Correspondencia: Carmen María Vizoso Gómez. Campus de Vegazana, s/n, 24071 León, España.

E-mail: cvizg@unileon.es 


\section{Introducción}

La resiliencia es un constructo que ha adquirido gran relevancia en el ámbito educativo debido al reciente interés por el estudio de los recursos y competencias asociadas al desarrollo saludable y el bienestar, tanto de educadores como de estudiantes (Boldrini, Sappa y Aprea, 2019; De la Fuente, López-García, Mariano-Vera, Martínez-Vicente y Zapata, 2017; RodríguezFernández, Ramos-Díaz, Ros y Zuazagoitia, 2018).

El concepto de resiliencia hace referencia a un proceso dinámico a través del cual se logra un crecimiento adaptativo después de afrontar de forma eficaz situaciones estresantes, desafíos y, sobre todo, tras superar las adversidades (Connor y Davidson, 2003; Masten, 2018; Rutter, 2007). Además, la resiliencia es una característica propia del desarrollo evolutivo y, por tanto, puede observarse en todas las personas en mayor o menor medida (Masten, 2018). No obstante, los hombres suelen presentar mayores niveles de resiliencia que las mujeres (Martínez-Martí y Ruch, 2017; Tomyn y Weinberg, 2018).

Tradicionalmente, se ha asociado la resiliencia con conceptos como resistencia, adaptación y capacidad de recuperación (Becoña, 2006). De igual modo, actualmente se defiende que la resiliencia guarda una estrecha relación con la salud, el bienestar y la satisfacción con la vida (Arrogante, 2014; Hu, Zhang y Wang, 2015; Sagone y De Caroli, 2014).

Dentro del ámbito educativo, Day y Gu (2015) defienden que la resiliencia es indispensable para mantener la calidad educativa, ya que los educadores resilientes están especialmente comprometidos con su trabajo y, además, promueven el desarrollo de la resiliencia en sus alumnos. Por su parte, Le Cornu (2009) apunta que la resiliencia es una fortaleza indispensable en los educadores, sobre todo durante los primeros años de ejercicio profesional. Además, la resiliencia protege la salud de los educadores porque ayuda a reducir la incidencia del burnout (Richards, Levesque-Bristol, Templin y Graber, 2016).

También se debe resaltar la relevancia de la resiliencia en el desarrollo de los estudiantes. Así, se ha encontrado que los estudiantes universitarios con altos niveles de resiliencia presentan también altos niveles de autorregulación, eficacia académica y compromiso con los estudios, además de bajos niveles de estrés y agotamiento (De la Fuente et al., 2017; Ríos-Risquez, Carrillo-García y Sabuco-Tebar, 2014; Ríos-Risquez, García-Izquierdo, Sabuco-Tebar, CarrilloGarcía y Martínez-Roche, 2016).

Recientemente se ha propuesto que la resiliencia está relacionada con el optimismo (Martínez-Martí y Ruch, 2017; Ramírez-Fernández, Ortega-Martínez y Calero-García, 2018). El optimismo se define como la predisposición a tener expectativas positivas hacia lo que sucederá en el futuro (Carver y Scheier, 2014). De este modo, las personas optimistas utilizan las estrategias necesarias para lograr sus objetivos porque esperan alcanzarlos, mientras que los pesimistas consideran que sus resultados serán siempre negativos. Además, los optimistas presentan mayores niveles de salud y bienestar que los pesimistas (Extremera, Durán y Rey, 2007; He, Cao, Feng, Guan y Peng, 2013; Segerstrom, Carver y Scheier, 2017).

Respecto al contexto educativo, se ha establecido que los educadores con un alto nivel de optimismo experimentan menores niveles de estrés laboral (Extremera, Durán y Rey, 2010). Del mismo modo, los estudiantes optimistas presentan bajos niveles de estrés, cansancio emocional y de depresión (Denovan y Macaskill, 2017; Hojat, Vergare, Isenberg, Cohen y Spandorfer, 2015). Además, se ha asociado el optimismo con el bienestar y la satisfacción con la vida en los 
estudiantes universitarios (Cazalla-Luna y Molero, 2016; Denovan y Macaskill, 2017). Sin embargo, los universitarios pesimistas suelen experimentar mayores niveles de afecto negativo y estrés (Ferrando, Chico y Tous, 2002; Tuckwiller y Dardick, 2018).

Por último, en la literatura científica se ha establecido una clara relación entre el optimismo y las estrategias activas de afrontamiento al estrés (Carver, Scheier y Segerstrom, 2010; Solberg Nes y Segerstrom, 2006; Rutter, 2007). Estas estrategias constituyen las posibles reacciones cognitivas y conductuales ante determinadas situaciones estresantes (Folkman y Lazarus, 1988; Lazarus y Folkman, 1984). Las principales estrategias activas de afrontamiento son la solución de problemas, la reestructuración cognitiva, la expresión de emociones y la búsqueda de apoyo social (Jauregui, Herrero-Fernández y Estévez, 2016; Tobin, Holroyd y Reynolds, 1984; Tobin, Holroyd, Reynolds y Wigal, 1989).

En estudios previos se ha demostrado que los profesores que utilizan estrategias de afrontamiento adecuadas muestran mayores niveles de autoeficacia y de bienestar laboral (Bermejo-Toro, Prieto-Ursúa y Hernández, 2016). Por otra parte, los universitarios que manejan estrategias activas de afrontamiento presentan un mayor compromiso con sus estudios y más optimismo (González-Cabanach, Souto-Gestal, González-Doniz y Franco-Taboada, 2018; Vizoso, Rodríguez y Arias-Gundín, 2018). Por último, se ha propuesto que el empleo de estrategias de afrontamiento como la solución de problemas y la reestructuración está relacionado con la resiliencia (Sagone y De Caroli, 2014).

En definitiva, tal y como se ha establecido previamente, la resiliencia supone la capacidad de adaptación frente a la adversidad y se asocia con la salud y el bienestar, tanto en educadores como en estudiantes universitarios. Por consiguiente, se considera de especial relevancia conocer cuáles son los factores que influyen en el desarrollo de la resiliencia entre los estudiantes de Ciencias de la Educación, que en un futuro próximo serán educadores. Para ello, en este estudio se plantean los siguientes objetivos:

(i) evaluar si existen diferencias estadísticamente significativas entre hombres y mujeres en resiliencia, optimismo, pesimismo y/o en las estrategias de afrontamiento activo,

(ii) analizar si el optimismo, el pesimismo y/o las estrategias de afrontamiento estudiadas se relacionan de forma significativa con la resiliencia para cada género, $y$

(iii) analizar si el optimismo, el pesimismo y las estrategias de afrontamiento activo predicen la resiliencia para cada género.

\section{Método}

\section{Participantes}

La muestra está formada por 450 estudiantes de la Universidad de León (España) matriculados en los grados de Educación Infantil (26.7\%), Educación Primaria (58.7\%) y Educación Social (14.7\%). Del total de participantes, 112 son hombres (24.9\%) y 338 son mujeres (75.1\%), con una media de edad de 20.34 años $(D T=2.03)$.

\section{Instrumentos}

Para medir la resiliencia se empleó la escala 10-item CD-RISC (Connor-Davidson Resilience Scale). La primera versión de la CD-RISC fue desarrollada por Connor y Davidson 
(2003) y constaba de 25 ítems. Posteriormente se publicó una versión reducida compuesta por 10 ítems (Campbell-Sills y Stein, 2007). En este estudio se empleó la versión reducida adaptada al español (Notario-Pacheco, Solera-Martínez, Serrano-Parra, Bartolomé-Gutiérrez, GarcíaCampayo y Martínez-Vizcaíno, 2011), que consta de 10 ítems (e.g. "Soy capaz de adaptarme a los cambios"). La escala de respuesta es de tipo Likert graduada de 0 (nunca) a 4 (siempre). Un alto nivel de resiliencia se corresponde con altas puntuaciones en la escala. La fiabilidad de la escala en este estudio es de $\alpha=.85$, idéntica a la obtenida en la versión en español (NotarioPacheco et al., 2011).

Para evaluar el optimismo se empleó el LOT-R (Life Orientation Test). La primera versión del LOT fue desarrollada por Scheier y Carver (1985). Posteriormente, se publicó una versión revisada denominada LOT-R (Scheier, Carver y Bridges, 1994). En este estudio se empleó la versión adaptada al español (Cano-García et al., 2015). El LOT-R consta de 10 ítems, de los cuales 3 están redactados en sentido positivo (e.g. "En tiempos difíciles, suelo esperar lo mejor"), 3 en sentido negativo (e.g. "Rara vez espero que las cosas salgan a mi manera") y 4 son de relleno para hacer menos evidente el contenido del test (e.g. "Me resulta fácil relajarme"). La escala de respuesta es de tipo Likert y oscila entre 0 (muy en desacuerdo) y 4 (muy de acuerdo). Las altas puntuaciones en los tres ítems positivos indican un alto optimismo y puntuaciones altas en los 3 ítems negativos indican un alto pesimismo. En este estudio, la fiabilidad del LOT-R es de $\alpha=.78$, similar a la descrita en la versión en español (Cano-García et al., 2015). Para la escala de optimismo la fiabilidad es de $\alpha==.77$ y para la escala de pesimismo es de $\alpha==.62$.

Para medir las estrategias de afrontamiento al estrés se empleó el CSI (Coping Strategies Inventory). La versión original del CSI fue desarrollada por Tobin et al. (1989) y consta de 40 ítems. Este instrumento presenta una estructura jerárquica y está formado por 2 escalas: Afrontamiento activo y Afrontamiento evasivo. En concreto, en este estudio se empleó la Escala de Afrontamiento Activo del CSI, en la versión adaptada al español por Jauregui et al. (2016). Esta escala consta de 4 subescalas: Solución de problemas (SP) o modificación de la situación que provoca el estrés (e.g. "Elaboré un plan de acción y lo seguí"), Reestructuración cognitiva (RC) o reinterpretación del significado de la situación estresante (e.g. "Me convencí de que las cosas no son tan malas como parecen"), Expresión de emociones (EE) o exteriorización de los sentimientos (e.g. "Dejé aflorar mis sentimientos") y Apoyo social (AS) o búsqueda de amparo emocional en otras personas (e.g. "Hablé con alguien acerca de cómo me estaba sintiendo"). La escala de respuesta tipo Likert oscila entre 1 (nunca utilizada) y 5 (siempre utilizada). La fiabilidad en este estudio para la subescala SP es de $\alpha=.77$, para RC es de $\alpha=.81$, para EE es de $\alpha=.68$ y para AS es de $\alpha=.88$. Para el total de la escala de Afrontamiento activo la fiabilidad es de $\alpha=.85$. La fiabilidad descrita por Jauregui et al. (2016) en la versión española era de $\alpha=.88$ para la subescala SP, $\alpha=.81$ para RC, $\alpha=.85$ para EE, $\alpha=.88$ para AS y $\alpha=.90$ para la escala de Afrontamiento activo.

\section{Procedimiento}

La información se recogió en la Facultad de Ciencias de Educación, donde cursan sus estudios todos los participantes en esta investigación. La técnica de recogida de información fue el cuestionario. Los cuestionarios se aplicaron en el aula habitual de los alumnos de forma grupal en una única sesión, contando con el consentimiento del profesor responsable. Todos los 
participantes recibieron instrucciones mediante las cuales se les indicaba los objetivos del estudio y la relevancia de responder a todos los enunciados con sinceridad. Además, se les indicó que la participación era voluntaria y que la información recogida era anónima y sería tratada de forma confidencial, respetándose los principios éticos fundamentales establecidos en la Declaración de Helsinki. Para resolver las dudas de los participantes, durante toda la sesión estaba presente una psicóloga experta.

\section{Resultados}

Las diferencias de género se analizaron mediante la prueba $t$ de Student para muestras independientes. El tamaño del efecto estadístico se evaluó con el valor $d$ de Cohen. En la tabla 1 se puede observar las diferencias entre hombres y mujeres para cada variable analizada.

Los resultados ponen de manifiesto que existen diferencias de género significativas en la variable resiliencia, presentando los hombres puntuaciones más altas que las mujeres. Siguiendo los criterios de Cohen (1988), el tamaño del efecto es pequeño $(d=.367)$.

Respecto a al optimismo, también se observan diferencias significativas, con puntuaciones más altas para los hombres, aunque el efecto es pequeño $(d=.224)$. Sin embargo, no existen diferencias significativas de género en la variable pesimismo.

En relación con las estrategias de afrontamiento, se observan diferencias de género significativas, siendo más altas las puntuaciones para las mujeres que para los hombres en la estrategia de expresión emocional, con un efecto pequeño $(d=.390)$, y la estrategia de apoyo social, con un efecto mediano $(d=.467)$.

Tabla 1. Estadísticos Descriptivos para la Muestra Total y para cada Género y Prueba T de Contraste de Medias para la Escala CD-RISC, las Subescalas del LOT-R y las Subescalas del CSI

\begin{tabular}{lccccccc}
\hline Instrumento & & $\begin{array}{c}\text { Total } \\
M(D T)\end{array}$ & $\begin{array}{c}\text { Hombres } \\
M(D T)\end{array}$ & $\begin{array}{c}\text { Mujeres } \\
M(D T)\end{array}$ & $T$ & $p$ & $d$ \\
\hline CD-RISC & RES & $2.72( \pm .64)$ & $2.89( \pm .61)$ & $2.66( \pm .64)$ & 10.537 & .001 & .367 \\
LOT-R & OPT & $2.45( \pm .95)$ & $2.61( \pm .91)$ & $2.40( \pm .96)$ & 4.151 & .042 & .224 \\
& PES & $2.02( \pm .84)$ & $2.00( \pm .84)$ & $2.03( \pm .84)$ & .119 & .730 & - \\
CSI & SP & $3.48( \pm .71)$ & $3.39( \pm .68)$ & $3.51( \pm .71)$ & 2.210 & .138 & - \\
& RC & $3.59( \pm .72)$ & $3.50( \pm .66)$ & $3.62( \pm .73)$ & 2.309 & .129 & - \\
& EE & $2.97( \pm .71)$ & $2.76( \pm .66)$ & $3.03( \pm .72)$ & 12.598 & .000 & .390 \\
& AS & $3.69( \pm .95)$ & $3.37( \pm .90)$ & $3.80( \pm .94)$ & 18.213 & .000 & .467 \\
\hline
\end{tabular}

Nota 1: RES = Resiliencia, OPT = Optimismo, PES = Pesimismo, $\mathrm{SP}=$ Solución de problemas, $\mathrm{RC}=$ Reestructuración cognitiva, $\mathrm{EE}=$ Expresión de emociones, $\mathrm{AS}=$ Apoyo social

Los coeficientes de correlación de Pearson entre la resiliencia y las demás variables se presentan en la tabla 2.

La resiliencia se relaciona de forma significativa y positiva con el optimismo, tanto si se considera la muestra total de participantes como al analizar hombres y mujeres por separado. Además, la correlación entre la resiliencia y el optimismo es la más alta para todas las muestras. Por el contrario, existe una relación significativa pero negativa entre la resiliencia y el pesimismo, observada también para todas las muestras. 
En cuanto a las estrategias de afrontamiento al estrés, se encontraron relaciones estadísticamente significativas y positivas entre la resiliencia y las estrategias de solución de problemas, reestructuración cognitiva y de apoyo social al analizar la muestra total y la muestra de mujeres. Por otra parte, en la muestra de hombres se observan relaciones significativas y positivas entre la resiliencia y las estrategias de solución de problemas y reestructuración cognitiva. Por último, la estrategia de expresión emocional no se relacionó de forma significa con la resiliencia para ninguna de las muestras.

Tabla 2. Correlaciones de Pearson entre la Resiliencia y las demás Variables para la Muestra Total y para cada Género

\begin{tabular}{lcccc}
\hline & & Total & Hombres & Mujeres \\
\hline Instrumento & Variables & RES & RES & RES \\
LOT-R & OPT & $.568^{* *}$ & $.585^{* *}$ & $.556^{* *}$ \\
& PES & $-.287^{* *}$ & $-.281^{* *}$ & $-.289^{* *}$ \\
CSI & SP & $.476^{* *}$ & $.442^{* *}$ & $.509^{* *}$ \\
& RC & $.454^{* *}$ & $.459^{* *}$ & $.475^{* *}$ \\
& EE & .029 & .096 & .044 \\
& AS & $.087^{*}$ & .092 & $.129^{* *}$ \\
\hline
\end{tabular}

Nota 1: RES $=$ Resiliencia, OPT $=$ Optimismo, $\mathrm{PES}=$ Pesimismo, $\mathrm{SP}=$ Solución de problemas, $\mathrm{RC}=$ Reestructuración cognitiva, $\mathrm{EE}=$ Expresión de emociones, $\mathrm{AS}=$ Apoyo social

Nota $2: * p<.05 ; * * p<.01$

El análisis de regresión lineal se realizó mediante el método de pasos sucesivos y se consideraron como posibles predictoras de la resiliencia aquellas variables que presentaron una correlación significativa con ella. Por lo tanto, para la muestra formada por los hombres se consideraron las variables optimismo, pesimismo, solución de problemas y reestructuración cognitiva, y para la muestra total y la formada por las mujeres se consideraron estas mismas variables, pero además se incluyó la variable apoyo social. En la tabla 3 se presentan los resultados de los análisis de regresión para cada muestra.

Los análisis de regresión lineal muestran que, tanto para la muestra total como para la muestra formada por hombres y la formada por mujeres, la variable que contribuye a la predicción de la resiliencia con mayor peso es el optimismo. La estrategia de afrontamiento de solución de problemas también presenta poder de predicción sobre la resiliencia para todas las muestras, pero con un peso menor. La varianza explicada por el modelo para cada una de las muestras fue superior al 40\%. No obstante, cabe destacar que en el caso de las mujeres el modelo alcanza a explicar significativamente más varianza que para los hombres. 
Tabla 3. Análisis de Regresión Lineal sobre la Resiliencia para la Muestra Total y para cada Género

\begin{tabular}{lccccccc}
\hline & $R$ & $R^{2}$ & $F$ & & $\beta$ & $t$ & $p$ \\
\hline Total & .653 & .427 & 166.390 & & & & \\
& & & & OPT & .468 & 12.490 & .000 \\
Hombres & .647 & .419 & 39.289 & & & & \\
& & & & OPT & .496 & 6.474 & .000 \\
& & & & SP & .291 & 3.790 & .000 \\
Mujeres & .661 & .436 & \multirow{2}{*}{129.666} & & & & \\
& & & & OPT & .442 & 10.265 & .000 \\
& & & & SP & .375 & 8.698 & .000 \\
\hline
\end{tabular}

Nota 1: Optimismo $=\mathrm{OPT}$, Solución de problemas $=\mathrm{SP}$

\section{Discusión}

El primer objetivo de este trabajo consistía en evaluar las diferencias entre los y las estudiantes de Ciencias de la Educación en cuanto a la resiliencia, el optimismo-pesimismo y las estrategias activas de afrontamiento. Los resultados revelan que existen diferencias de género significativas en relación con resiliencia. En concreto, los estudiantes presentan mayor resiliencia que las estudiantes. Los estudios previos ofrecían resultados contradictorios. Así, mientras que Campbell-Sills y Stein (2007) no hallaron diferencias significativas entre universitarios y universitarias, en los trabajos más recientes se establece que los hombres presentan una mayor resiliencia que las mujeres (Martínez-Martí y Ruch, 2017; Tomyn y Weinberg, 2018). En este sentido, Hu et al. (2015) explican que estas diferencias pueden deberse a los roles sociales de género que representan a las mujeres como el sexo débil y, por lo tanto, con menores niveles de resiliencia que los hombres. No obstante, es necesario realizar nuevos estudios para tratar de establecer evidencias más concluyentes sobre este resultado.

Asimismo, los resultados indican que existen diferencias de género significativas en relación con el optimismo, siendo los universitarios más optimistas que las universitarias. Estos resultados coinciden con los obtenidos por otros autores, que resaltan las diferencias en la socialización de hombres y mujeres como posible explicación a las diferencias encontradas (Cazalla-Luna y Molero, 2016; Extremera et al., 2007).

Además, los resultados indican que existen diferencias de género significativas en algunas estrategias de afrontamiento, con puntuaciones más altas para las mujeres que para hombres. También Tobin, et al. (1984) encontraron que las universitarias empleaban las estrategias activas en mayor medida que los universitarios. Recientemente se ha propuesto que estas diferencias están relacionadas con el empoderamiento de las mujeres que se está promoviendo a través de los movimientos feministas (Wilsey y Lyke, 2015).

Por otra parte, en este estudio se pretendía analizar la relación de la resiliencia con el optimismo-pesimismo y con las estrategias de afrontamiento. En primer lugar, la relación entre resiliencia y optimismo-pesimismo observada en este estudio coincide con los resultados descritos en la literatura científica previa (Martínez-Martí y Ruch, 2017; Ramírez-Fernández et 
al., 2018). Así, se considera que las personas más optimistas presentan mayor resiliencia y las personas pesimistas muestran menor resiliencia. En segundo lugar, se encuentra que la resiliencia se asocia con estrategias de afrontamiento como la solución de problemas y la reestructuración cognitiva, pero no se relaciona con la estrategia de expresión emocional. Previamente, Sagone y De Caroli (2014) establecieron también una clara asociación entre resiliencia y estrategias de afrontamiento en estudiantes universitarios.

Por otra parte, los análisis de regresión señalan la relevancia de la solución de problemas como estrategia que predice la resiliencia; es decir, los estudiantes que afrontan activamente las dificultades, tratando de solventar los problemas, consiguen superarlas y logran además un desarrollo adecuado. Del mismo modo, otros autores han defendido que la estrategia con mayor poder de predicción sobre la resiliencia en los jóvenes es la solución de problemas (CampbellSills, Cohan y Stein, 2006). Por último, los resultados del presente estudio indican que el optimismo es el factor que presenta un mayor poder de predicción sobre la resiliencia en todos los estudiantes participantes. Por tanto, los y las estudiantes con expectativas positivas respecto al futuro conseguirán superar las adversidades. En la misma línea, se ha descrito el optimismo como predictor de la resiliencia en las personas que han vivido situaciones muy traumáticas (Segovia, Moore, Linnville, Hoyt y Hain, 2012).

En definitiva, se puede concluir que el optimismo y la solución de problemas para afrontar el estrés tienen poder de predicción sobre la resiliencia en los estudiantes universitarios de Ciencias de la Educación, independientemente de las diferencias que se puedan dar entre ambos géneros en cada una de las variables analizadas. Estos datos aportan información que puede ser relevante para el diseño de programas de intervención que tengan como objetivo el desarrollo de la resiliencia en los futuros educadores. De esta forma, teniendo en cuenta que tanto el optimismo como las estrategias de afrontamiento son recursos que determinan la resiliencia y que pueden ser adquiridos y optimizados (Herrero et al, en prensa; Malouff y Schutte, 2017), se debería incluir actuaciones para su entrenamiento y potenciar así el desarrollo de la resiliencia.

\section{Referencias}

Arrogante, O. (2014). Mediación de la resiliencia entre burnout y salud en el personal de Enfermería. Enfermería Clínica, 24(5), 283-289. doi: 10.1016/j.enfcli.2014.06.003

Becoña, E. (2006). Resiliencia: definición, características y utilidad del concepto. Revista de Psicopatología y Psicología Clínica, 11(3), 125-146.

Bermejo-Toro, L., Prieto-Ursúa, M., y Hernández, V. (2016). Towards a model of teacher wellbeing: personal and job resources involved in teacher burnout and engagement. Educational Psychology, 36(3): 481-501. doi: 10.1080/01443410.2015.1005006

Boldrini, E., Sappa, V., y Aprea, C. (2019). Which difficulties and resources do vocational teachers perceive? An exploratory study setting the stage for investigating teachers' resilience in Switzerland. Teachers and Teaching, 25(1), 125-141. doi: 10.1080/13540602.2018.1520086

Campbell-Sills, L., Cohan, S. L., y Stein, M. B. (2006). Relationship of resilience to personality, coping, and psychiatric symptoms in young adults. Behaviour Research and Therapy, 44(4), 585-599. doi: 10.1016/j.brat.2005.05.001 
Campbell-Sills, L., y Stein, M.B. (2007). Psychometric analysis and refinement of the ConnorDavidson Resilience Scale (CD-RISC): Validation of a 10-item measure of resilience. Journal of Traumatic Stress, 20, 1019-1028. doi: 10.1002/jts.20271

Cano-García, F. J., Sanduvete-Chaves, S., Chacón-Moscoso, S., Rodríguez-Franco, L., GarcíaMartínez, J., Antuña-Bellerín, M. A., y Pérez-Gil, J. A. (2015). Factor structure of the Spanish version of the Life Orientation Test-Revised (LOT-R): Testing several models. International Journal of Clinical and Health Psychology, 15, 139-148. doi: 10.1016/j.ijchp.2015.01.003

Carver, C.S., y Scheier, M. (2014). Dispositional optimism. Trends in Cognitive Sciences, 18, 293-299. doi: 10.1016/j.tics.2014.02.003

Carver, C. S., Scheier, M. F., y Segerstrom, S. C. (2010). Optimism. Clinical Psychology Review, 30, 879-889. doi: 10.1016/j.cpr.2010.01.006.

Cazalla-Luna, N., y Molero, D. (2016). Inteligencia emocional percibida, disposición al optimismo-pesimismo, satisfacción vital y personalidad de docentes en su formación inicial. Revista de Investigación Educativa, 34(1), 241-258. doi: 10.6018/rie.34.1.220701

Cohen, J. (1988). Statistical power analysis for the behavioral sciences. Hillsdale, NJ: Erlbaum.

Connor, K., y Davidson, J. (2003). Development of a new resilience scale: The ConnorDavidson Resilience Scale (CD-RISC). Depression and Anxiety, 18, 76-82. doi: 10.1002/da.10113

Day, C., y Gu, Q. (2015). Educadores resilientes, escuelas resilientes: construir y sostener la calidad educativa en tiempos dificiles. Madrid: Narcea.

De la Fuente, J., López-García, M., Mariano-Vera, M., Martínez-Vicente, J. M., y Zapata, L. (2017). Autorregulación personal, enfoques de aprendizaje, resiliencia y ansiedad evaluativa en estudiantes de Psicología. Estudios sobre Educación, 32, 9-26. doi: 10.15581/004.32.9-26

Denovan, A., y Macaskill, A. (2017). Stress and subjective well-being among first year UK undergraduate students. Journal of Happiness Studies, 18(2), 505-525. doi: 10.1007/s10902-016-9736-y

Extremera, N., Durán, A., y Rey, L. (2007). Perceived emotional intelligence and dispositional optimism-pessimism: Analyzing their role in predicting psychological adjustment among adolescents. Personality and Individual Differences, 42, 1069-1079. doi: 10.1016/j.paid.2006.09.014

Extremera, N., Durán, A., y Rey, L. (2010). Recursos personales, síndrome de estar quemado por el trabajo y sintomatología asociada al estrés en docentes de enseñanza primaria y secundaria. Ansiedad y Estrés, 16, 47-60.

Ferrando, P. J., Chico, E., y Tous, J. M. (2002). Propiedades psicométricas del test de optimismo Life Orientation Test. Psicothema, 14, 673-680.

Folkman, S., y Lazarus, R. S. (1988). Coping as a mediator of emotion. Journal of Personality and Social Psychology, 54(3), 466-475. doi:10.1037/0022-3514.54.3.466

González-Cabanach, R., Souto-Gestal, A., González-Doniz, L., y Franco-Taboada, V. (2018). Perfiles de afrontamiento y estrés académico en estudiantes universitarios. Revista de Investigación Educativa, 36(2), 421-433. doi: 10.6018/rie.36.2.290901 
He, F., Cao, R., Feng, Z., Guan, H., y Peng, J. (2013). The impacts of dispositional optimism and psychological resilience on the subjective well-being of burn patients: A Structural Equation Modelling Analysis. PLoS ONE 8(12), e82939. doi: 10.1371/journal.pone.0082939

Herrero, R., Mira, A., Cormo, G., Etchemendy, E., Banos, R., García-Palacios, A., ... Botella, C. (en prensa). An Internet based intervention for improving resilience and coping strategies in university students: Study protocol for a randomized controlled trial. Internet Interventions. doi: 10.1016/j.invent.2018.03.005

Hojat, M., Vergare, M., Isenberg, G., Cohen, M., y Spandorfer, J. (2015). Underlying construct of empathy, optimism, and burnout in medical students. International Journal of Medical Education. 6, 12-16. doi: 10.5116/ijme.54c3.60cd

Hu, T., Zhang, D., y Wang, J. (2015). A meta-analysis of the trait resilience and mental health. Personality and Individual Differences, 76, 18-27. doi: 10.1016/j.paid.2014.11.039

Jauregui, P., Herrero-Fernández, D., y Estévez, A. (2016). Estructura factorial del "Inventario de Estrategias de Afrontamiento" y su relación con la regulación emocional, ansiedad y depresión. Psicología Conductual, 24(2), 319-340.

Lazarus, R. S., y Folkman, S. (1984). Stress, appraisal, and coping. New York: Springer.

Le Cornu, R. (2009). Building resilience in pre-service teachers. Teaching and Teacher Education, 25(5), 717-723. doi: 10.1016/j.tate.2008.11.016

Malouff, J. M., y Schutte, N. S. (2017). Can psychological interventions increase optimism? A meta-analysis. The Journal of Positive Psychology, 12(6), 594-604. doi: 10.1080/17439760.2016.1221122

Martínez-Martí, M.L., y Ruch, W. (2017). Character strengths predict resilience over and above positive affect, self-efficacy, optimism, social support, self-esteem, and life satisfaction. The Journal of Positive Psychology, 12, 110-119. doi: 10.1080/17439760.2016.1163403

Masten, A. S. (2018). Resilience theory and research on children and families: Past, present, and promise. Journal of Family Theory \& Review, 10, 12-31. doi: 10.1111/jftr.12255

Notario-Pacheco, B., Solera-Martínez, M., Serrano-Parra, M., Bartolomé-Gutiérrez, R., GarcíaCampayo, J., y Martínez-Vizcaíno, V. (2011). Reliability and validity of the Spanish version of the 10-item Connor-Davidson Resilience Scale (10-item CD-RISC) in young adults. Health and Quality of Life Outcomes, 9, 63-69. doi: 10.1186/1477-7525-9-63

Ramírez-Fernández, E., Ortega-Martínez, A. R., y Calero-García, M. J. (2018). El optimismo como mediador entre la resiliencia y estados afectivos en adultos mayores. Estudios de Psicología, 39(2-3), 275-285. doi: 10.1080/02109395.2018.1486360

Richards, K. A. R., Levesque-Bristol, C., Templin, T. J., y Graber, K. C. (2016). The impact of resilience on role stressors and burnout in elementary and secondary teachers. Social Psychology of Education, 19(3), 511-536. doi: 10.1007/s11218-016-9346-x

Ríos-Risquez, M. I., Carrillo-García, C., y Sabuco-Tebar, E. L. (2014). Resiliencia, síndrome de quemarse por el trabajo y malestar psicológico en estudiantes de enfermería. Ansiedad y Estrés, 20(2-3), 115-126.

Ríos-Risquez, M. I., García-Izquierdo, M., Sabuco-Tebar, E. L., Carrillo-García, C., y MartínezRoche, M. E. (2016). An exploratory study of the relationship between resilience, academic burnout and psychological health in nursing students. Contemporary Nurse, 52, 430-439. doi: 10.1080/10376178.2016.1213648 
Rodríguez-Fernández, A., Ramos-Díaz, E., Ros, I., y Zuazagoitia, A. (2018). Implicación escolar de estudiantes de secundaria: La influencia de la resiliencia, el autoconcepto y el apoyo social, percibido. Educación XX1, 21(1), 87-108. doi: 10.5944/educXX1.16026

Rutter, M. (2007). Resilience, competence, and coping. Child Abuse \& Neglect, 31, 205-209. doi: $10.1016 / j$.chiabu.2007.02.001

Sagone, E., y De Caroli, M. E. (2014). A Correlational Study on dispositional resilience, psychological well-being, and coping strategies in university students. American Journal of Educational Research, 2(7), 463-471. doi: 10.12691/education-2-7-5

Scheier, M. F., y Carver, C. S. (1985). Optimism, coping, and health: Assessment and implications of generalized outcome expectancies. Health Psychology, 4, 219-247. doi: 10.1037/0278-6133.4.3.219

Scheier, M. F., Carver, C. S., y Bridges, M. W. (1994). Distinguishing optimism from neuroticism (and trait anxiety, self-mastery, and self-esteem): A reevaluation of the life orientation test. Journal of Personality and Social Psychology, 67, 1063-1078. doi: 10.1037//0022-3514.67.6.1063

Segerstrom, S. C., Carver, C. S., y Scheier, M. F. (2017). Optimism. En M. D. Robinson y M. Eid (Eds.), The happy mind Cognitive contributions to well-being. (pp. 195-212). Cham, Switzerland: Springer International Publishing. doi: 10.1007/978-3-319-58763-9_11

Segovia, F., Moore, J. L., Linnville, S. E., Hoyt, R. E., y Hain, R. E. (2012). Optimism predicts resilience in repatriated prisoners of war: a 37-year longitudinal study. Journal of Traumatic Stress, 25(3), 330-336. doi: 10.1002/jts.21691

Solberg Nes, L., y Segerstrom, S. C. (2006). Dispositional optimism and coping: A meta-analytic review. Personality and Social Psychology Review, 10(3), 235-251. doi: 10.1207/s15327957pspr1003_3

Tobin, D.L., Holroyd, K.A., y Reynolds, R.V.C. (1984). Users manual for Coping Strategies Inventory. Ohio: University Press.

Tobin, D. L., Holroyd, K. A., Reynolds, R. V. C., y Wigal, J. K. (1989). The hierarchical factor structures of the Coping Strategies Inventory. Cognitive Therapy and Research, 13(4), 343-361. doi: 10.1007/BF01173478

Tomyn, A., y Weinberg, M. (2018). Resilience and subjective wellbeing: A psychometric evaluation in young Australian adults. Australian Psychologist, 53(1), 68-76. doi: 10.1111/ap.12251

Tuckwiller, B., y Dardick, W. R. (2018). Mindset, grit, optimism, pessimism, and life satisfaction in university students with and without anxiety and/or depression. Journal of Interdisciplinary Studies in Education, 6(2), 32-48.

Vizoso, C., Rodríguez, C., y Arias-Gundín, O. (2018). Coping, academic engagement and performance in university students. Higher Education Research \& Development, 37(7), 1515-1529. doi: 10.1080/07294360.2018.1504006

Wilsey, C., y Lyke, J. A. (2015). Gender differences in perceived illness, stress, and coping in undergraduates. Psychology Research, 5(3), 189-196. doi: 10.17265/2159$5542 / 2015.03 .005$ 